\title{
Can the Planck Length Be Found Independent of Big G?
}

\author{
Espen Gaarder Haug ${ }^{1}$
}

${ }^{1}$ Norwegian University of Life Sciences, Norway

Correspondence: Espen Gaarder Haug, Norwegian University of Life Sciences, Norway. E-mail: espenhaug@mac.com

Received: May 22, 2017

doi:10.5539/apr.v9n6p58
Accepted: June 24, 2017

Online Published: November 12, 2017

URL: https://doi.org/10.5539/apr.v9n6p58

\begin{abstract}
In this paper we show how it is possible to measure the Planck length through a series of different methods. One of these measurements is totally independent of big G, but moving from the theoretical realm to the empirical realm would require particle accelerators far more powerful than the ones that we have today. However, a Cavendish-style experiment can also be performed to find the Planck length with no knowledge of the value of big G. Furthermore, the Cavendish style set-up gives half of the relative measurement error in the Planck length compared to the measurement error in big G.
\end{abstract}

Keywords: Planck length, independent of big G, maximum velocity, Cavendish-style experiment, gravity, orbital velocity, gravitational red-shift, deflection of light, Planck constant, speed of light, mass

\section{Introduction and Challenge}

The Planck length was first introduced by Max Planck in 1906, see (Planck, 1906), and is given as

$$
l_{p}=\sqrt{\frac{\hbar G}{c^{3}}} \approx 1.616229 \times 10^{-35} \text { meter }
$$

This shows the Planck length as a function of Newton's (1686) big G, the reduced Planck constant, and the speed of light. Haug (2016a, 2016b, 2016c) has recently suggested that big $G$ is a universal composite constant that can be written in the form

$$
G=\frac{l_{p}^{2} c^{3}}{\hbar}
$$

Using this formula for big $\mathrm{G}$ simplifies and quantifies a long series of equations in Newton's and Einstein's conception of gravity. It has recently come to our attention that (McCulloch, 2014) has derived a similar formula for big $\mathrm{G}$ based on Heisenberg's uncertainty principle (Heisenberg, 1927)

$$
G=\frac{\hbar c}{m_{p}^{2}}
$$

Since $m_{p}=\frac{\hbar}{l_{p}} \frac{1}{c}$ the (McCulloch, 2014) and the (Haug, 2016a) formulas are basically the same

$$
G=\frac{\hbar c}{m_{p}^{2}}=\frac{\hbar c}{\left(\frac{\hbar}{l_{p}} \frac{1}{c}\right)^{2}}=\frac{l_{p}^{2} c^{3}}{\hbar} .
$$

Formula (4) can naturally be found by simply rewriting the Planck length formula (1) with respect to big G. However, (Haug, 2016b) has also derived this formula from dimensional analysis as well as from Heisenberg's uncertainty principle, using his newly-introduced maximum velocity formula for matter (Haug, 2017). McCulloch has derived his formula from Heisenberg's uncertainty principle as well, but relies on a very different method. The argument in favor of writing big $\mathrm{G}$ in this way is grounded in the fact that it helps us quantize and simplify a long series of formulas from Einstein's and Newton's gravitational theories without changing their values. 
Both of these proposed formulas (Haug and McCulloch) for big G may be criticized for appearing to lead to circular arguments that have no solution, at least at first glance. Until recently, the Planck length has only been known to be found by using big G. From this perspective, the Planck length seems to be a derived constant from the more fundamental constant, big G. Therefore, it may not seem sound to claim that big G can be a function of the Planck length. Here we will challenge this view by pointing out several ways of finding the Planck length independently of knowing big $\mathrm{G}$.

\section{The Planck Length Totally Independent of Big G}

Haug (2016d, 2016e, 2016f, 2017) has suggested that there may be a maximum velocity for matter just below the speed of light given by

$$
v_{\max }=c \sqrt{1-\frac{l_{p}^{2}}{\bar{\lambda}^{2}}}
$$

This formula can be derived by assuming that the reduced Compton wavelength can never be shorter than the Planck length, or that the maximum frequency we can observe on relativistic Doppler shift is the Planck frequency. The formula has also been derived from the recent progress in mathematical atomism. Haug (2014) has shown that all of Einstein's special relativity mathematical end results can be derived from atomism when using EinsteinPoincarè synchronized clocks. He assumes that the diameter of the smallest possible fundamental particle is the Planck length and that this particle makes up all energy and matter; this must not be confused with the standard view of particles.

Assuming that this is the maximum velocity of anything with rest-mass also seems to solve a series of infinity challenges in modern physics. It gives a limit on the maximum kinetic energy for a subatomic particle, as well as a maximum on the momentum and on the proper velocity for anything with rest-mass. Haug has further shown that the absence of such a speed limit leads to absurd possibilities, such as a single electron with a relativistic mass equal to that of the Sun, or even an entire galaxy. If a single electron like this ever hit the Earth, the Earth would be pulverized. Clearly, this has not happened in the billions of years the Earth has existed and indicates that there must be an exact upper boundary on the velocity on the electron that falls below the speed of light.

The maximum velocity formula given by Haug can be solved with respect to the Planck length.

$$
l_{p}=\bar{\lambda} \sqrt{1-\frac{v_{\max }^{2}}{c^{2}}}
$$

The reduced Compton wavelength of an electron can also be found independently of big G, see (Prasannakumar, Krishnaveni, \& Umesh, 2012). Vmax had to be observed experimentally to find the Planck length.

Calculations show that this maximum velocity for any known observed subatomic particle (such as an electron) is just below the speed of light, but far above the rate that has been attained for particle acceleration in the Large Hadron Collider (LHC), for example. In other words, this new way of observing the Planck length is only a theory at this time. However, if we assume that this represents the maximum velocity of anything containing matter, then we will find that a series of infinity's challenges in physics will disappear, see (Haug, 2016f).

Furthermore, recent developments in mathematical atomism strongly strengthen our suspicion that the Planck length is one of the most fundamental constants. All of Einstein's special relativity equations and a series of additional equations have been derived simply by assuming that everything consists of indivisible particles always traveling at the speed of light in the void (empty space). First, when linking the diameter of this particle with the Planck length we are able to get the mass of the electron consistent with the reduced Compton wavelength of the electron under a theory derived from atomism. Atomism is also one of several ways to calculate the maximum velocity of anything with rest-mass.

\section{Planck Length from a Cavendish-Style Set-Up}

We can easily set up a Cavendish-style (Cavendish, 1798) experiment (even with a low budget) and find the Planck length without direct knowledge of big G. Further, we need no knowledge of the mass of the Earth or any other cosmological objects. The Cavendish experiments consist of first finding the weight of four balls, two small ones and two large ones. These balls can consist of any element, but here we will use an example of lead balls. The mass of these balls can be found by taking a weight at the surface of the Earth, or one could use the Watt Balance, see (Stock, 2011), for example. The two small lead balls are placed at a distance of $L$ apart on a rod that we will hang on a wire. We leave the two heavier lead balls in a stationary position next to each of the smaller lead balls. 
For a full understanding of the set-up, we recommend reading about the Cavendish experiment; see Cavendish 1798 , for the original description; many other sources are available in books and online.

We will call the distance between centers of the large and small balls (when the balance is deflected) $r$. The period of oscillation of torsion balance is measured as T. If we have an accurate estimate of the Planck constant and know the value of the speed of light ${ }^{1}$ through other experiments, we can now directly find the Planck length from the Cavendish-style experiment by using the following formula

$$
l_{p}=\sqrt{\frac{\hbar 2 \pi^{2} L r^{2} \theta}{M T^{2} c^{3}}}
$$

where $G$ is the angle in radians of deflection of the torsion balance beam from its rest position. (See Appendix A for a full derivation of this formula when not relying on big $\mathrm{G}$ at all). This is basically the same experiment that Cavendish performed. Cavendish did not actually calculate big G, but used his experiment to find the density of the Earth and thereby determine the weight of the Earth. One could imagine that the Planck constant and the speed of light had been measured and were well-known before anyone had figured out the value of big G.

In such a case, one could come up with the gravitational formula $F=\frac{l_{p}^{2} c^{3}}{\hbar} \frac{M M}{r^{2}}$ without knowing big $\mathrm{G}$ and the unknown we would be searching for would be the Planck length and not big G.

Before the development of Newton's theory of gravitation, if one assumed that gravity had to travel with the speed of light then it would be natural to think that one needed to include the speed of light somehow in the formula for gravity. Now, in our view, the speed of light is embedded even within Newton's formula inside big G.

We can actually measure the Planck length from the kitchen table using a small size Cavendish-style set-up, likely with less than a 5\% error. One can build such an apparatus for a few dollars in materials, or one can buy a readyto-use commercial "home-kit" for a few thousand dollars. A small-size armature Cavendish-style set-up can measure big G to an accuracy of $\pm 10 \%$ or better (depending on the apparatus), and the Planck length to an accuracy that is twice as good at that.

\section{The Error in the Newton Gravitational Constant Is Twice the Measurement Error in the Planck Length}

To measure the gravitational constant and the Planck length is, in many ways, two sides of the same coin, particularly under the view that Newton's gravitational constant is a composite constant that is a function of the Planck length. When using any form of gravitational measurement to find the Planck length, we will see that it looks like the measurement error in Newton's gravitational constant should be about twice the error of the measurement in the Planck length.

The partial derivative of big $\mathrm{G}$ with respect to the Planck length is given by

$$
\frac{\partial G}{\partial l_{p}}=\frac{2 c^{3} l_{p}}{\hbar}
$$

In terms of percentage sensitivity in $\mathrm{G}$ with respect to the percentage error in the Planck length, we must have

$$
\% \text { Sensitivity }=\frac{2 c^{3} l_{p}}{\hbar} \frac{l_{p}}{G \times 100}=\frac{2 \frac{l_{p}^{2} c^{3}}{\hbar}}{G \times 100}=\frac{2}{100}
$$

That is for a $1 \%$ error in the measurement in the Planck length, we get about a $2 \%$ error in the measurement of the gravitational constant. Further, we can partially derive the Planck formula for the Planck length with respect to big $\mathrm{G}$, and we get

$$
\frac{\partial l_{p}}{\partial G}=\frac{\hbar}{2 c^{3} \sqrt{\frac{G \hbar}{c^{3}}}}
$$

This gives us the percentage sensitivity in the Planck length for a percentage point change (error) in big G

$$
\% \text { Sensitivity }=\frac{\sqrt{\hbar}}{2 \sqrt{G c^{3}}} \frac{G}{l_{p} \times 100}=\frac{\sqrt{\frac{G \hbar}{c^{3}}}}{l_{p} \times 200}=\frac{1}{200}
$$


That is to say, for each one percent error in the measurement of Newton's gravitational constant we only have about half a percent error in the measurement of the Planck length. One could argue that for the Planck length we have uncertainty in the Planck constant that will affect the uncertainty of the Planck length. This is true, but the uncertainty in the Planck constant is almost negligible. If we take into account three standard deviations in the uncertainty of the Planck constant ${ }^{2}$ (from 2014 CODATA), we get a percentage error in the Planck length of only $\pm 0.00002304 \%$. The one standard deviation relative error is only about $-7.68084199413574 \times 10^{-08}$. This is negligible compared to the known relative standard error in the Planck length.

We can also indirectly see from the CODATA 2014 reported standard errors that the measurement error (as measured in percentage of the constant) is twice as large for Newton's gravitational constant as it is for the Planck length. For big G, the CODATA reports a standard error of $0.00031 \times 10^{-11} \mathrm{~m}^{3} \mathrm{~kg}^{-1} \mathrm{~s}^{-2}$, in percentage of the gravitational constant that is a relative standard error of $4.65 \times 10^{-05}$. And for the Planck length, the CODATA reports a standard error of $0.000038 \times 10^{-35}$ and a relative standard error of $2.3 \times 10^{-5}$. It is no coincidence that the relative standard error in the Planck length is basically half of the standard error for the gravitational constant; it comes from the relationship between them.

\section{The Planck Length from Orbital Velocity}

We can also find the Planck length from orbital velocity. The orbital velocity is given by

$$
\begin{gathered}
v_{o}=\sqrt{\frac{G M}{r}} \\
v_{o}=\sqrt{\frac{\frac{l_{p}^{2} c^{3}}{\hbar} M}{r}} \\
v_{o}=\sqrt{\frac{l_{p}^{2} c^{3} M}{\hbar r}}
\end{gathered}
$$

Solved with respect to the Planck length we get

$$
l_{p}=\sqrt{\frac{v_{0}^{2} \hbar r}{c^{3} M}}
$$

We can find the Planck length from knowing the orbital velocity of a satellite. This again would require knowledge of the mass of the Earth (or the mass we are measuring orbital velocity around). We could easily find the mass of the Earth by performing the Cavendish experiment and finding the Planck length before calculating big G based on the Planck length, the Planck constant, and the speed of light.

Assume a human-made satellite is orbiting the Earth at an altitude of $600 \mathrm{~km}$ and at a measured orbital velocity of $7561.36 \mathrm{~m} / \mathrm{s}$. Since the radius of the Earth is about $6,371 \mathrm{~km}$, this gives a radius of the satellite (relative to the center of the mass it is orbiting) of 6,971,000 meters. The mass of the Earth is $5.972 \times 10^{24} \mathrm{~kg}$. This gives us the input to find the Planck length

$$
l_{p}=\sqrt{\frac{v_{0}^{2} \hbar r}{c^{3} M}}=\sqrt{\frac{7561.36^{2} \times \hbar \times 6,971,000}{c^{3} \times 5.972 \times 10^{24}}} \approx 1.6162 \times 10^{-35} \mathrm{~m}
$$

Again one can argue that we need to know big G to know the mass of the Earth and that we are entering in a circular argument. However, the bottom line here is that we can find the Planck length, the Planck constant, and the speed of light independent of any knowledge of big G. Further, the mass of fundamental particles can be found simply by knowing their reduced Compton wavelength, which can be found by Compton scattering.

\section{The Planck Length from the Gravitational Acceleration Field}

We can also find the Planck length from the gravitational acceleration field.

$$
l_{p}=r \sqrt{\frac{g \hbar}{c^{3} M}}
$$

For Earth, the gravitational acceleration field at the surface is about $9.807 \mathrm{~m} / \mathrm{s}^{2}$. From this plus the radius and mass of the Earth we know the Planck length

$$
l_{p}=6371000 \sqrt{\frac{9.807 \times \hbar}{c^{3} \times 5.972 \times 10^{24}}} \approx 1.61519 \times 10^{-35}
$$




\section{The Planck Length from Gravitational Light Deflection}

By assuming $G=\frac{l_{p}^{2} c^{3}}{\hbar}$ we can rewrite Einstein's gravitational light deflection formula

$$
\begin{gathered}
\delta=\frac{4 G M}{c^{2} r} \\
\delta=\frac{4 \frac{l_{p}^{2} c^{3}}{\hbar} M}{c^{2} r} \\
\delta=\frac{4 l_{p}^{2} M c}{\hbar r}
\end{gathered}
$$

This we can solve with respect to $l_{p}$, which gives us

$$
l_{p}=\sqrt{\frac{\hbar r \delta \frac{\pi}{648000}}{4 M c}}
$$

where $\delta_{\text {is }}$ the observed bending of light in arcseconds, $r$ is the radius from the center of the mass bending on the light to the point at which the light passes the object, $M$ is the mass of the object, $\mathrm{c}$ is the speed of light, and $\hbar$ is the reduced Planck constant.

To give an example: for the Sun, the observed light bending is 1.75 arcseconds or $1.75 / 3600$ of a degree. The radius of the sun is $696,342,000$ meters and the mass of the Sun is $M_{S} \approx 1.98810^{30} \mathrm{~kg}$. We can plug this into the formula above and obtain

$$
l_{p}=\sqrt{\frac{\hbar r_{s} \delta_{s} \frac{\pi}{648000}}{4 M_{s} c}}=\sqrt{\frac{\hbar \times 696342000 \times 1.75 \times \frac{\pi}{648000}}{4 \times 1.988 \times 10^{30} \times c}} \approx 1.6162 \times 10^{-35} \mathrm{~m}
$$

\section{The Planck Length from Gravitational Red-Shift}

Gravitational deflection is hard to measure very accurately. The technology used to measure gravitational red-shift is (likely) much more accurate. This involves gravitational time dilation that can be measured with very accurate optical clocks today. In a weak gravitational field (like we have on the Earth and that also exists on the surface of the Sun) we have

$$
\begin{aligned}
& \lim _{r \rightarrow+\infty} z(r) \approx \frac{2 G M}{c^{2} r} \\
& \lim _{r \rightarrow+\infty} z(r) \approx \frac{2 \frac{l_{p}^{2} c^{3}}{\hbar} M}{c^{2} r} \\
& \lim _{r \rightarrow+\infty} z(r) \approx \frac{2 l_{p}^{2} M c}{\hbar r}
\end{aligned}
$$

Solved with respect to the Planck length we get

$$
l_{p}=\sqrt{\frac{\hbar r z(r)}{2 M c}}
$$

We could even measure the gravitational red-shift between two different altitudes on the surface of the Earth, on smaller sized objects like the Moon, or even onboard a large spherical space station. For the gravitational red-shift measured from two different radius related to the same mass (object), we have the following formula that works very well in weak gravitational fields 


$$
\begin{gathered}
\frac{\lambda_{2}-\lambda_{1}}{\lambda_{1}} \approx \frac{1+\frac{2 G M}{c^{2} \eta}}{1+\frac{2 G M}{c^{2} \eta_{2}}}-1 \\
\frac{\lambda_{2}-\lambda_{1}}{\lambda_{1}} \approx \frac{2 \frac{l_{p^{c}}^{2}}{\hbar} M}{1+\frac{c^{2} \eta}{l^{2} c^{3}}}-1 \\
\frac{2 \frac{\frac{l^{c}}{\hbar} M}{c^{2} r_{2}}}{\lambda_{2}-\lambda_{1}} \\
\lambda_{1} \approx \frac{1+\frac{2 \frac{l_{p}^{2}}{\hbar} M}{\eta}}{1+\frac{2 \frac{l_{p}^{2}}{\hbar} M}{\eta^{c}}}-1
\end{gathered}
$$

Solved with respect to the Planck length we get

$$
l_{p}=\sqrt{\frac{\frac{\lambda_{2}-\lambda_{1}}{\lambda_{1}} \hbar r_{1} r_{2}}{2 c M r_{2}-2 c M r_{1}-2 \frac{\lambda_{2}-\lambda_{1}}{\lambda_{1}} c M r_{1}}}
$$

In other words, we can find the Planck length simply from gravitational red-shift observations, the mass of the object, the reduced Planck constant, and the speed of light.

\section{Table Summary}

In the table below we show a series of formulas related to different types of measurements that can be used to measure the Planck length

Table 1. Ways to Find the Planck Length

\begin{tabular}{lll}
\hline Ways to find the Planck length & Planck length formulas & Comments \\
\hline Traditional Max Planck Formula & $l_{p}=\sqrt{\frac{G \hbar}{c^{3}}}$ & Directly dependent on big G. \\
$\begin{array}{l}\text { Maximum velocity of subatomic } \\
\text { particles }\end{array}$ & $l_{p}=\bar{\lambda} \sqrt{1-\frac{v_{\max }^{2}}{c^{2}}}$ & $\begin{array}{l}\text { No need for big G in calculation, } \\
\text { but } v_{\max } \text { above current } \\
\text { accelerators. } \\
\text { Cavendish-style experiment }\end{array}$ \\
$\begin{array}{ll}\text { Can be done from kitchen table. } \\
\text { Gravital velocity }\end{array}$ & $l_{p}=\sqrt{\frac{\hbar 2 \pi^{2} L r^{2} \theta}{M T^{2} c^{3}}}$ & Easy to do from Earth's surface. \\
Gravitational red-shift & $l_{p}=\sqrt{\frac{v_{0}^{2} \hbar r}{c^{3} M}}$ & Easy to do from Earth's surface. \\
Gravitational deflection & $l_{p}=\sqrt{\frac{\hbar r z(r)}{2 M c}}$ & Easy to do from Earth's surface. \\
& $l_{p}=\sqrt{\frac{\hbar r \delta}{4 M c}}$ & Solar deflection observed from \\
\hline
\end{tabular}

Note. In the table above we show a series of formulas related to different types of measurements that can be used to measure the Planck length elsewhere in the article. 


\section{Conclusion}

We have shown how the Planck length can be found through a number of methods including a Cavendish-style experiment, the orbital velocity, the gravitational acceleration field, the gravitational red-shift, and the gravitational deflection. To do this we need to know the mass of the object, the reduced Planck constant, the speed of light, and the radius related to the measurements. We have also shown that one can find the Planck length from the newlyintroduced maximum velocity of something with rest-mass.

The notation in Newton's gravitational constant offers a hint that it is a universal composite constant rather than a fundamental constant. It makes sense when we have meters and time, that the Planck length is the shortest length unit that can ever be measured. The speed of light is the fastest rate at which something can travel and it consists of distance divided by time. The Newton gravitational constant is in the form $\mathrm{m}^{3} \mathrm{~kg}^{-1} \mathrm{~s}^{-2}$ - it seems unlikely that anything at the very deepest level should be meters cubed divided by $\mathrm{kg}$ and seconds squared.

The gravitational constant is a composite (derived) constant, while the Planck length represents something physical; it is the shortest reduced Compton wavelength possible. According to recent developments in mathematical atomism, there are also strong indications that the Planck length is the diameter of the only truly fundamental particle, namely an indivisible particle that together with void is making up all matter and energy, see (Haug, 2014, 2016f).

\section{Acknowledgments}

Thanks to Victoria Terces for helping me edit this manuscript and thanks to Mike McCulloch and Eric Thompson for useful comments.

\section{References}

Cavendish, H. (1798). Experiments to determine the density of the Earth. By Henry Cavendish, Esq. FRS and AS. Philosophical Transactions of the Royal Society of London, 88, 469-526.

Haug, E. G. (2017). A Suggested Boundary for Heisenberg's Uncertainty Principle. Retrieved from http://vixra.org/abs/1701.0497

Haug, E. G. (2014). Unified Revolution: New Fundamental Physics. Oslo: E.G.H. Publishing.

Haug, E. G. (2016a). Planck Quantization of Newton and Einstein Gravitation. International Journal of Astronomy and Astrophysics, 6(02), 206.

Haug, E G. (2016b). The gravitational constant and the Planck units. A simplification of the quantum realm. Physics Essays, 29(4), 558-561.

Haug, E. G. (2016c). Planck Quantization of Newton and Einstein Gravitation for Planck Masses and Smaller Size Objects. Retrieved from www.viXra.org 1610.03282017

Haug, E. G. (2016d). A New Solution to Einstein's Relativistic Mass Challenge Based on Maximum Frequency. Retrieved from http://vixra.org/abs/1609.0083

Haug, E. G. (2016e). Deriving the Maximum Velocity of Matter from the Planck Length Limit on Length Contraction. Retrieved from http://vixra.org/abs/1612.0358, 2016

Haug, E. G. (2016f). The Planck Mass Particle Finally Discovered! Good bye to the Point Particle Hypothesis! Retrieved from http://vixra.org/abs/1607.0496

Haug, E. G. (2017). The ultimate limits of the relativistic rocket equation. The Planck photon rocket. Acta Astronautica, 136, 144-147.

Haug, E. G. (2017b). Newton's E =mc ${ }^{2}$ Two Hundred Years Before Einstein? Newton = Einstein at the Quantum Scale. Retrieved from http://vixra.org/abs/1706.0371

Heisenberg, W. (1927). Über den Anschaulichen Inhalt der Quantentheoretischen Kinematik und Mechanik. Zeitschrift für Physik, 43.

McCulloch, M. E. (2014). Gravity from the Uncertainty Principle. Astrophysics and Space Science, 349(2).

Newton, I. (1686). Philosophiae Naturalis Principia Mathematica. London.

Planck, M. (1906). The Theory of Radiation. Dover 1959 translation.

Prasannakumar, S., Krishnaveni, S., \& Umesh, T. K. (2012). Determination of Rest Mass Energy of the Electron by a Compton Scattering Experiment. European Journal of Physics, 33(1). 
Stock, M. (2011). The Watt Balance: Determination of the Planck Constant and Redefinition of the Kilogram. Philosophical Transactions of the Royal Society of London, 369.

\section{Notes}

Note 1 . The speed of light is exact by definition.

Note 2. CODATA 2014 gives a Planck constant of $6.626070040 \times 10^{-34}$, and a one standard error of 0.000000081 $\times 10^{-34}$.

\section{Appendix A: The Planck Length Found Directly from a Cavendish Apparatus}

From a Cavendish apparatus one can surprisingly find the Planck length directly without any knowledge of big G. A Cavendish apparatus consist of two small balls (made of lead, for example) and two larger balls. The torque is given by

$$
\kappa \theta
$$

where $\kappa$ is the torsion coefficient of the suspending wire and $\theta$ is the deflection angle of the balance. We have the following well-known relationship

$$
\kappa \theta=L F
$$

where $L$ is the length between the two small balls in the apparatus and $F$ is the gravitational force given by the Newton formula, but also by the alternative gravitational constant-less formula, see (Haug, 2017b)

$$
F=G \frac{M m}{r^{2}}=\frac{M c^{2}}{r} \frac{m}{m_{p}} \frac{l_{p}}{r}
$$

One could easily imagine that someone had suggested the last part of formula (23) as a formula for gravity instead of the Newtonian version with a gravitational constant. In the last part of formula (23), however, we have what would be the unknown Planck length and the Planck mass; could we find them without any reliance on big G?

From equation (22) and (23) we must have

$$
\kappa \tau=L \frac{M c^{2}}{r} \frac{m}{m_{p}} \frac{l_{p}}{r}
$$

Further, the natural resonant oscillation period of the torsion balance is given by

$$
T=2 \pi \sqrt{\frac{I}{\kappa}}
$$

The moment of inertia $I$ of the balance is given by

$$
I=m\left(\frac{L}{2}\right)^{2}+m\left(\frac{L}{2}\right)^{2}=2 m\left(\frac{L^{2}}{2}\right)
$$

This means we have

$$
T=2 \pi \sqrt{\frac{m L^{2}}{2 \kappa}}
$$

And solved with respect to $\kappa$ this gives

$$
\begin{aligned}
& \frac{T^{2}}{2^{2} \pi^{2}}=\frac{m L^{2}}{2 \kappa} \\
& \kappa=\frac{m L^{2}}{2 \frac{T^{2}}{2^{2} \pi^{2}}} \\
& \kappa=\frac{m L^{2} 2 \pi^{2}}{T^{2}}
\end{aligned}
$$

Next we are replacing this expression for $\kappa$ into equation (24) and solving with respect to the Planck length 


$$
\begin{gathered}
\frac{m L^{2} 2 \pi^{2}}{T^{2}} \theta=L \frac{M c^{2}}{r} \frac{m}{m_{p}} \frac{l_{p}}{r} \\
\frac{m L^{2} 2 \pi^{2}}{T^{2}} \theta=L \frac{M c^{2}}{r} \frac{m}{\frac{\hbar}{l_{p}} \frac{1}{c}} \frac{l_{p}}{r} \\
\frac{\hbar L 2 \pi^{2} r^{2}}{M c^{3} T^{2}} \theta=l_{p}^{2} \\
l_{p}=\sqrt{\frac{\hbar L 2 \pi^{2} r^{2} \theta}{M c^{3} T^{2}}}
\end{gathered}
$$

Be aware that $M$ here is the mass of the large lead balls in the Cavendish apparatus, not the mass of the Earth. All we need to find the mass of the large balls is an accurate weight. The Planck constant can be measured by a Watt Balance experiment, for example, and the speed of light is given. The point is that we can find the Planck length with no knowledge of the gravitational constant.

After we have found the Planck length, we can find the mass of the Earth by measuring the gravitational acceleration of an object and then using the formula

$$
M=\frac{g r^{2} \hbar}{l_{p}^{2} c^{3}}
$$

From this we can easily calculate the orbital velocity of the Moon around the Earth, or any other gravitational calculation we are interested in. Our approach has the strength that the Planck length is something we can logically understand. It is a length and likely is linked to the smallest relevant length in the universe. On the other hand, what is the gravitational constant, this exotic animal that is meters cubed divided by $\mathrm{kg}$ and seconds squared $\left(\mathrm{m}^{3}\right.$ $\left.\mathrm{kg}^{-1} \mathrm{~s}^{-2}\right)$ ? It is difficult to get any intuition from the gravitational constant alone, except from understanding that it is a constant we can measure empirically and use to get Newton's formula to match actual observations. By looking at it as a composite constant, we can break it down into its more fundamental parts, all of which can be measured without big G.

In fact, we need no knowledge of big $\mathrm{G}$ to do any of the gravitational calculations. The smallest of all lengths is far too small to be seen, even using the most advanced electron microscope. Suprisingly, the smallest of all lengths can easily be measured from the kitchen table using a miniature Cavendish experiment plugged into a computer. In our wonderful modern age, ancient mechanical technology like the Cavendish apparatus can be combined with high-precision electronics to measure milliradians and time data that are fed straight into the computer and then the Planck length is revealed at last.

\section{Copyrights}

Copyright for this article is retained by the author(s), with first publication rights granted to the journal.

This is an open-access article distributed under the terms and conditions of the Creative Commons Attribution license (http://creativecommons.org/licenses/by/4.0/). 